\title{
Spontaneous and Ion-Specific Formation of Inverted Bilayers at Air/Aqueous Interface
}

\author{
Srikanth Nayak, Raju R. Kumal, and Ahmet Uysal*
}

Chemical Sciences and Engineering Division, Argonne National Laboratory, Lemont, IL 60439

KEYWORDS

Liquid-liquid extraction, Langmuir monolayers, inverted bilayers, lanthanide separation, ion-specific effects, amphiphile aggregation, vibrational sum frequency generation, ion adsorption 
Amphiphilic lipid-ion interactions at aqueous interfaces drive the assisted ion transport in various biological and industrial systems. In chemical separations of heavy elements, lipids coordinate metal ions and solubilize them in an organic phase. Direct observation of lipid-metal interactions is highly difficult at the buried oil/water interface, and is accessible with limited experiments. Here, we demonstrate that inverted bilayer structures previously observed at oil/aqueous interfaces can also be formed at the air/aqueous interface. This facilitates the easier study of lipid-ion interactions over a wide range of parameters with multiple probes, including synchrotron X-ray reflectivity (XR), X-ray fluorescence near total reflection (XFNTR), and vibrational sum-frequency generation spectroscopy (VSFG). The formation of bilayers is highly sensitive to the metal ion charge density. While $\mathrm{Lu}^{3+}(115$ $\left.\mathrm{C} / \mathrm{mm}^{3}\right)$ lead to bilayer formation, $\mathrm{Nd}^{3+}\left(82 \mathrm{C} / \mathrm{mm}^{3}\right)$ and $\mathrm{Sr}^{2+}\left(33 \mathrm{C} / \mathrm{mm}^{3}\right)$ lead to monolayers. By introducing $\mathrm{Lu}^{3+}$ ions to preformed lipid monolayers, we extract kinetic parameters corresponding to monolayer to inverted bilayer conversion. Temperature-dependent studies show Arrhenius behavior with an energy barrier of $40 \mathrm{kcal} / \mathrm{mol}$. The kinetics of monolayer to inverted bilayer conversion is also affected by the presence of background salts where thiocyanate accelerates the conversion more than nitrate does. Our results show the outsized importance of ion-specific effects on interfacial structure and kinetics, pointing to their role in chemical separation methods. Finally, this model system can be used to study a wide variety of lipid-ion interactions, opening a new avenue in molecular-scale understanding of these important systems. 


\section{INTRODUCTION}

Liquid-liquid extraction (LLE) is a chemical separation process widely used in hydrometallurgical processing of minerals, nuclear waste, and in recycling. ${ }^{1}$ Despite being used for decades, molecular-scale details of LLE are not well-understood. A clear identification of free energy drivers in LLE will lead more efficient separation technologies, a crucial task due to the increasing demand in rare earths, platinum group metals, and other critical materials.

LLE is a two-phase multicomponent equilibria driven process involving complex ionic equilibria in both the phases, interfacial effects, and solvent reorganization. ${ }^{2-3}$ The selective interfacial transport of metallic ions is thought to be mainly driven by the amphiphilic extractant molecules used in LLE. ${ }^{4}$ Thus, model systems focused on the interfacial interactions of ions with amphiphilic molecules have been used to elucidate the processes in LLE. ${ }^{5-14}$

Dialkyl phosphoric acid extractants have been widely used in LLE of metals, particularly rare earth elements. ${ }^{15} \mathrm{Bis}$ (2-ethylhexyl) phosphoric acid (HDEHP) preferentially extracts the heavier lanthanides in a dimeric form such that there are six phosphate groups coordinating the extracted lanthanide in the organic phase.${ }^{16}$ Reverse micellar structures are formed in the organic phase after extraction of metals with HDEHP. ${ }^{17-20}$ Interfacial studies using vibrational sum-frequency generation spectroscopy (VSFG) have shown the importance of hydrogen bonding interactions between the phosphate groups and water in stabilizing these micellar structures at the liquid-liquid interface. ${ }^{21}$ Peak shifts in VSFG in the phosphate region induced by different lanthanides have been attributed to increase in interaction strength between HDEHP and the lanthanides with increasing atomic number. ${ }^{22}$ Interestingly, interfacial $X$-ray scattering and fluorescence results suggest a preference for lighter lanthanides at the Gibbs monolayers of HDEHP at the air/aqueous interface, due to the higher solubility of HDEHP complexes of heavier lanthanides. ${ }^{23}$

Water-insoluble molecules, with longer alkyl tails, are limited to interface and can plausibly provide more information on the ion-extractant interactions. Dihexadecylphosphate (DHDP) forms an insoluble monolayer on water, and it has been used as an analogue of HDEHP for this purpose. ${ }^{6,14,24}$ Even then, the observation of extractant-metal complexes at the oil-water interface was not trivial. At the dodecane-water interface, extraction of $\mathrm{Er}^{3+}$ and $\mathrm{Y}^{3+}$ ions started at increased temperatures and then the system was cooled down to "arrest" inverted bilayer structures at the dodecane-water interface ${ }^{7,24}$ Although static structures of the inverted bilayers were observed in this arrested form, the limitations of the dodecane-water interface did not allow further investigations into the kinetics of the process. A similar experiment investigated dodecane-water interface in the presence of $\mathrm{Sr}^{2+}$ ions and observed a simple monolayer formation. The role of the ion size was suggested to cause the difference, but this could not be proven as divalent $\mathrm{Sr}^{2+}$ and trivalent $\mathrm{Er}^{3+}$ have multiple differences in addition to the ion size.

Langmuir monolayers at air-water interface are convenient model systems for LLE and have been well studied to probe intermolecular forces. ${ }^{5,25}$ The physical state of the interface depends on the intermolecular forces between the monolayer molecules and those between the monolayer and the subphase. Influence of dissolved chemical species on monolayers has been studied in order to understand the intermolecular interactions in various biological and physicochemical systems. ${ }^{26-29}$ 
Changes in the physical state of the interface are typically monitored by surface pressure isotherms, $\mathrm{X}$ ray and neutron scattering techniques, vibrational spectroscopy, and light microscopy techniques. ${ }^{25,29-32}$

Here, we show that the inverted bilayers of DHDP can be created at air/aqueous interface, similar to those formed at the oil/aqueous interface but more accessible for detailed investigations, using synchrotron X-ray scattering techniques and VSFG. We first show that the inverted bilayers form with $\mathrm{Lu}^{3+}$ but not with $\mathrm{Nd}^{3+}$, chemically very similar lanthanides, which shows that the charge density is the main driver behind the inverted bilayer formation. We also show that the bilayers can form regardless of the monolayer spreading procedure. When DHDP is spread on $\mathrm{Lu}^{3+}$ containing solutions, inverted bilayers form immediately. When DHDP monolayer is spread on pure water and $\mathrm{Lu}^{3+}$ ions are introduced later, the monolayer transforms into an inverted bilayer spontaneously. Temperature dependent studies determined that the monolayer to inverted bilayer transition follows an Arrhenius behavior. We also investigated the effects of background salts with nitrate and thiocyanate ions on the monolayer to inverted bilayer transition.
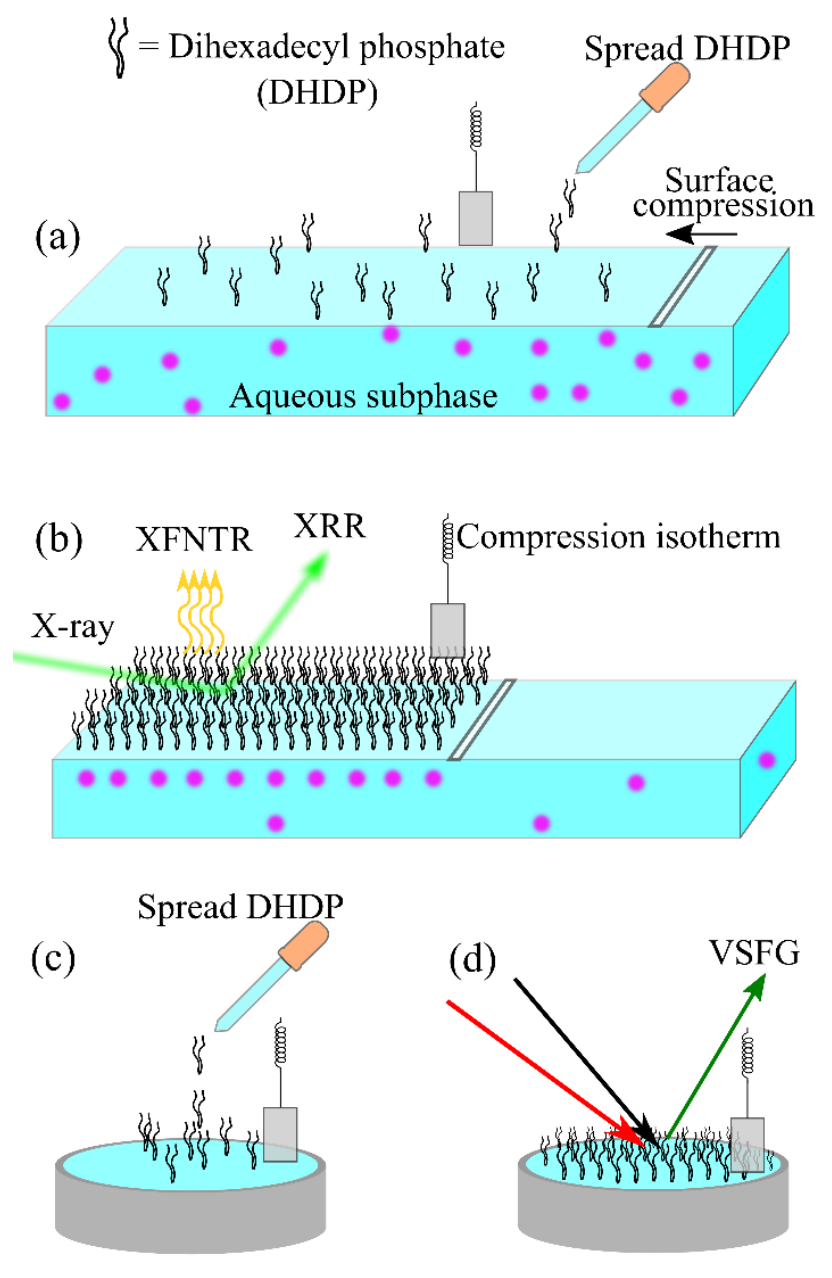

Figure 1. Schematic depiction of interaction of trivalent ions at air/aqueous interfaces and the analytical tools we have used in this study - surface pressure isotherms, X-ray reflectivity, X-ray fluorescence near total reflection, and VSFG. ( $a, b)$ For $X$-ray experiments, a subphase containing lanthanide ions is poured 
into the Langmuir trough and DHDP molecules are spread on the surface. A barrier in contact with the surface is moved to vary the surface pressure detected with a Wilhelmy plate. (c, d) For VSFG experiments the lanthanide containing solution was in a petri dish and DHDP molecules were spread from a more dilute solution.

\section{RESULTS AND DISCUSSIONS}

The effect of ions on Langmuir monolayers can be studied in two ways - a) monolayer molecules are spread on the electrolyte solution with ions of interest and compressed to reach a fixed surface pressure, or b) monolayer molecules are spread on pure water and compressed, followed by introduction of the ions in subphase. We have studied the effect of trivalent lanthanide ions on negatively charged DHDP monolayers using both methods. First, we describe the results obtained by directly spreading DHDP molecules on solutions of lanthanides (Figure 1).

\section{Spreading of DHDP on lanthanide solutions}
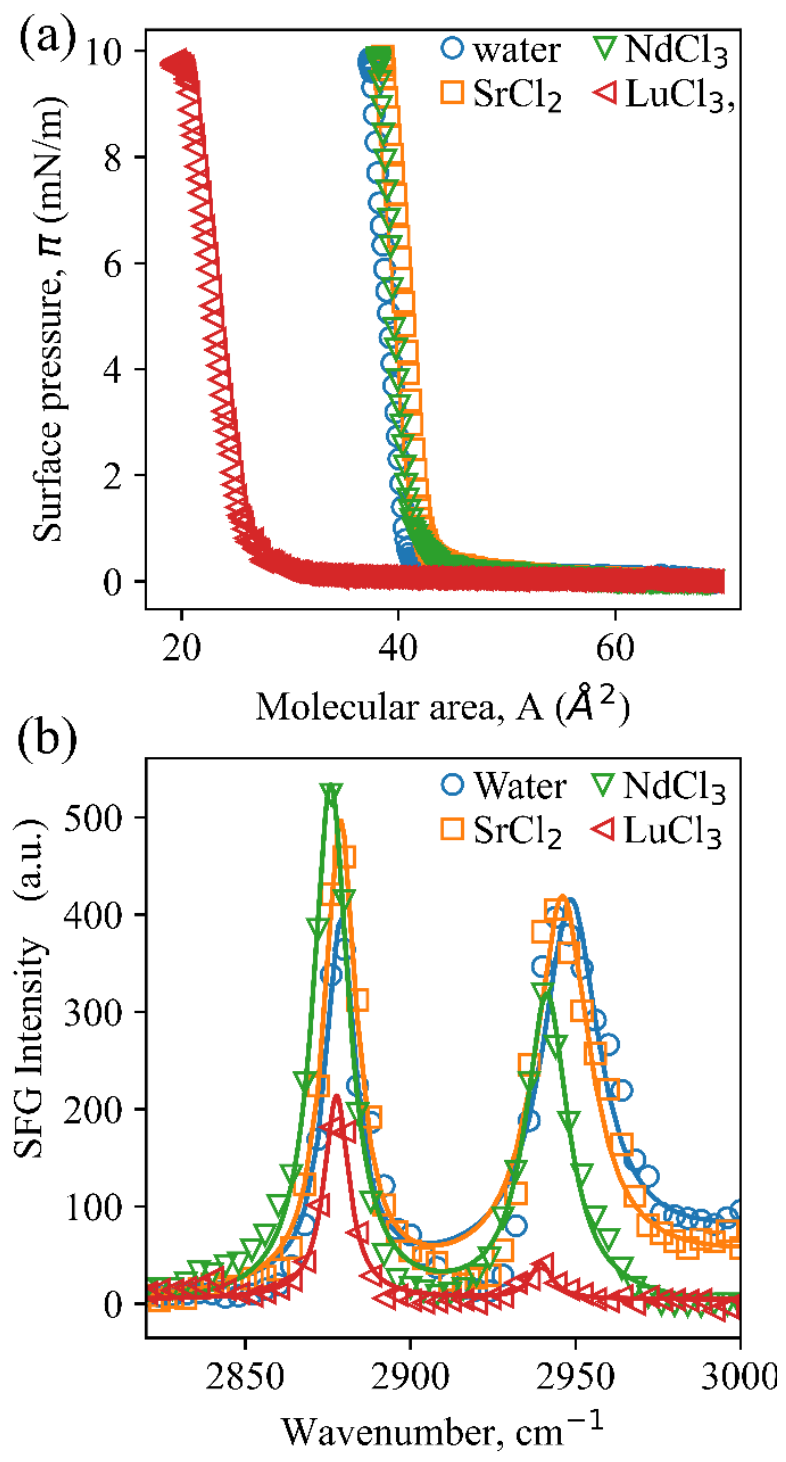
Figure 2. a) Langmuir isotherms showing the formation of monolayers of DHDP on surface of water (blue), $0.1 \mathrm{mM} \mathrm{SrCl}_{2}$ (orange), $0.1 \mathrm{mM} \mathrm{NdCl}_{3}$ (green), and inverted bilayer with $0.1 \mathrm{mM} \mathrm{LuCl}_{3}$. (b) VSFG results for the corresponding sample surfaces showing the difference in $\mathrm{CH}_{2}, \mathrm{CH}_{3}$ region spectra. The measurements were done in SSP polarization combination. The lower peak intensity of the $\mathrm{CH}_{3}$ region with $\mathrm{Lu}^{3+}$ compared to $\mathrm{Nd}^{3+}$ shows the increased symmetry at the interface in the presence of $\mathrm{Lu}^{3+}$.

Surface compression isotherms of Langmuir monolayers can provide information on phase transitions in the surfactant organization at the surface and the surface area occupied by surfactant molecules. ${ }^{25}$ Figure 2(a) shows the isotherms obtained by the compression of DHDP-covered surfaces in the presence of various ions. In the presence of $\mathrm{Sr}^{2+}$ or $\mathrm{Nd}^{3+}$ ions, the isotherms overlap with that obtained in the absence of any added ions. The surface pressure increases rapidly as the surface is compressed to $~ 40$ $\AA^{2}$ per DHDP. This is typical for a monolayer of double-chain molecule, considering that the molecular area for a single hydrocarbon tail is $20 \AA^{2}$. In contrast, in the presence of $\mathrm{Lu}^{3+}$ in the subphase, the surface pressure begins to rise at much lower values of surface area per DHDP. Given that the lowest area per DHDP for a closely packed monolayer is $\sim 40 \AA^{2}$, the lower area per DHDP $\left(\sim 27 \AA^{2}\right)$ in the presence of $\mathrm{Lu}^{3+}$ suggests the formation of bilayer or multilayer at the surface.

The average orientation of DHDP molecules would differ significantly when they are in monolayer versus bilayer or multilayer configuration. We use VSFG spectroscopy to probe the orientational distribution of DHDP molecules on the surfaces of the ionic solutions. The technique is non-destructive and inherently surface specific due to symmetry restriction and provides information on orientational ordering of the interfacial molecules. ${ }^{31,33}$ Figure 2(b) shows the VSFG spectra in the methyl and methylene stretch regions for DHDP-covered surfaces on different ionic solutions under SSP polarization configuration (where, $\mathrm{S}=\mathrm{SFG}, \mathrm{S}=$ visible, and $\mathrm{P}=\mathrm{IR}$ polarizations). The corresponding spectra in the $\mathrm{OH}$ region is shown in Figure $\mathrm{S} 1$ which provides the interfacial water structure. In the presence of $\mathrm{Nd}^{3+}$ or $\mathrm{Lu}^{3+}$, the $\mathrm{OH}$-band disappears which is generally observed when ions shied the monolayer surface charge and reduce charge-induced alignment of interfacial water molecules. ${ }^{8,34}$ This is in contrast to the presence of $\mathrm{OH}$ signal when metal hydroxide ions adsorb to the monolayer. ${ }^{35}$

A typical organic monolayer shows multiple resonances in this region corresponding to symmetric and asymmetric stretches of terminal $\mathrm{CH}_{3}$ groups $\left(2875 \mathrm{~cm}^{-1}\right.$ and $2960 \mathrm{~cm}^{-1}$, respectively), Fermi resonance between symmetric stretch and bending mode of $\mathrm{CH}_{3}\left(2940 \mathrm{~cm}^{-1}\right)$, and symmetric and asymmetric stretches of the $\mathrm{CH}_{2}$ groups on the tail $\left(2850 \mathrm{~cm}^{-1}\right.$ and $2920 \mathrm{~cm}^{-1}$, respectively). ${ }^{36}$ In Figure 2 (b) all the spectra show two prominent peaks centered around $2875 \mathrm{~cm}^{-1}$ and $2960 \mathrm{~cm}^{-1}$. The absence of any peak or shoulder in the $\mathrm{CH}_{2}$ stretch regions indicates that the tail groups are well-ordered at the surface for all the ionic conditions considered. ${ }^{36}$ The amplitude of the $\mathrm{CH}_{3}$ symmetric stretch vibration increases in the presence of $\mathrm{Sr}^{2+}$ and $\mathrm{Nd}^{3+}$ but decreases in the presence of $\mathrm{Lu}^{3+}$, relative to corresponding amplitude in the absence of ions. The adsorption of ions to DHDP reduces the electrostatic repulsion between the headgroups and realigns the tail groups vertically. The increase in the amplitude in SSP polarization is likely due to the realignment of DHDP tail groups in the presence of $\mathrm{Sr}^{2+}$ or $\mathrm{Nd}^{3+}$. The decrease in amplitude in the presence of $\mathrm{Lu}^{3+}$ is in correspondence with the formation of bi- or multilayers of DHDP. The ratio of amplitudes for $\mathrm{Nd}^{3+}$ and $\mathrm{Lu}^{3+}$ is $\sim 1 / 3$ which indicates the formation of a bilayer or an inverted bilayer, since the signal from the oppositely aligned $\mathrm{CH}_{3}$ groups cancel each other. However, VSFG data alone cannot determine whether the structure is a regular bilayer or an inverted bilayer. 

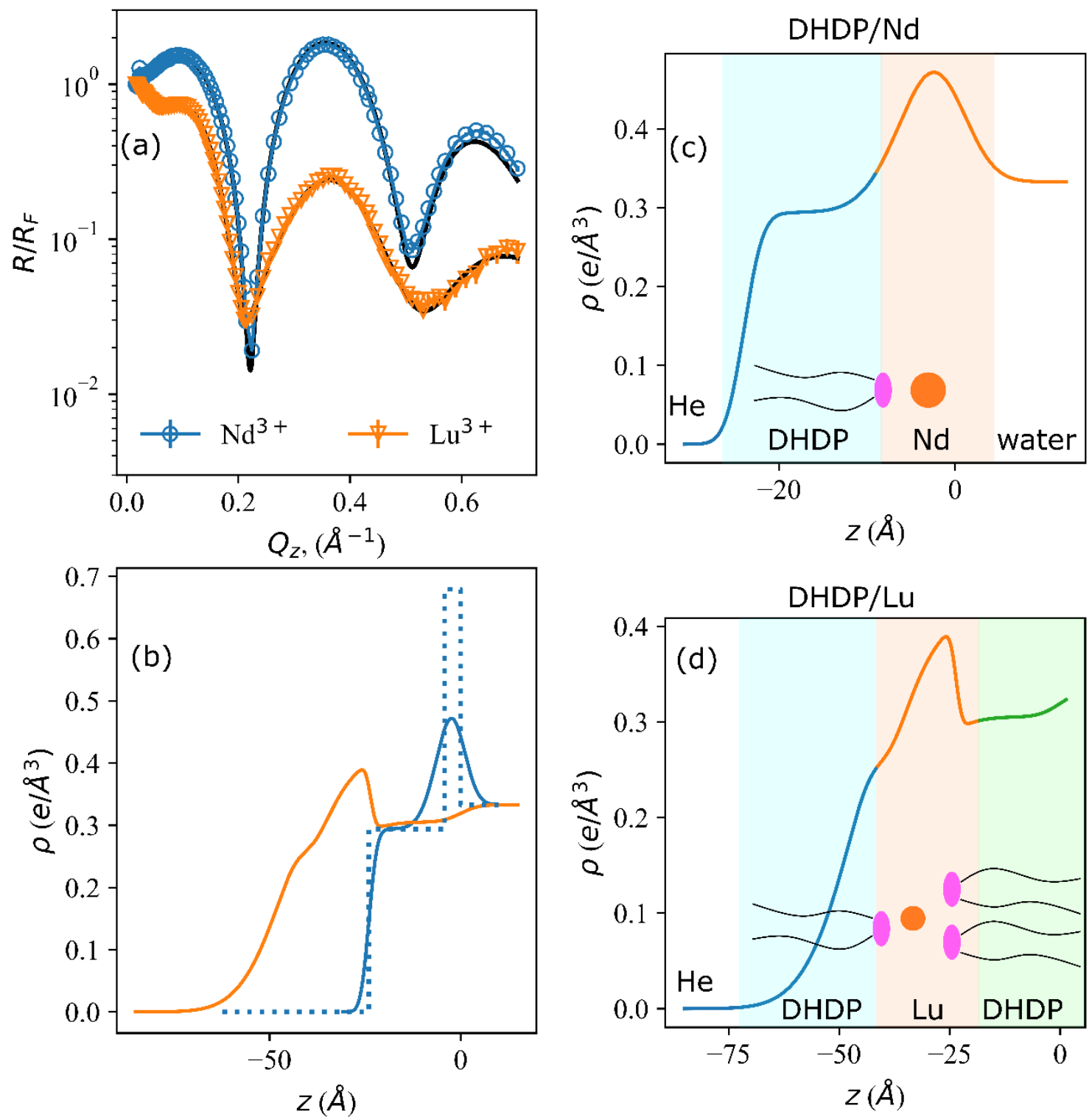

Figure 3. a) X-ray reflectivity results for $0.1 \mathrm{mM} \mathrm{NdCl}_{3}$ (blue circles) and $0.1 \mathrm{mM} \mathrm{LuCl}_{3}$ in water (orange squares) and the corresponding electron density profiles in (b). The fits for $\mathrm{NdCl}_{3}$ were obtained using a 2-box model corresponding to the monolayer tail group and the headgroup region. Blue dashed lines show the 2-box model fits for $\mathrm{Nd}^{3+}$ without roughness terms. The fits for $\mathrm{LuCl}_{3}$ were obtained with a 3box model and it shows the formation of an inverted bilayer at the air/aqueous interface. Schematics of the interfacial structure with $\mathrm{Nd}^{3+}$ and $\mathrm{Lu}^{3+}$ are shown in (c) and (d).

The interfacial structure can be resolved by specular X-ray reflectivity (XR) as it provides information on the electron density distribution normal to the surface. ${ }^{37-38}$ Figure 3(a) shows the Fresnel-normalized XR obtained for DHDP-covered surfaces in the presence of $\mathrm{Nd}^{3+}$ and $\mathrm{Lu}^{3+}$. There are major qualitative differences in the reflectivity curves - with $\mathrm{Nd}^{3+}$ the normalized reflectivity rises at low $\mathrm{Q}_{z}$ whereas it decreases in the presence of $\mathrm{Lu}^{3+}$. Further, the first extremum occurs at a smaller $\mathrm{Q}_{z}$ for $\mathrm{Lu}^{3+}$ surfaces than that for the $\mathrm{Nd}^{3+}$ surfaces. The electron density profile obtained from the box model fits to the 
reflectivity curves is shown in Figure 3(b). The fit parameters are given in the Table S2 which provide information on the thickness of the layer (d), electron density ( $\rho)$, and roughness ( $\sigma)$. The interfacial region is wider in the case of $\mathrm{Lu}^{3+}$ compared to that of $\mathrm{Nd}^{3+}$, which matches with the corresponding lower period of oscillations in the reflectivity curve. The electron density (ED) profile for $\mathrm{Nd}^{3+}$ sample neatly follows the expected profile for a monolayer, similar to those obtained with $\mathrm{La}^{3+} .{ }^{39}$ The boxes used in the fit according to the expected physical properties of the system - tail group region with a thickness of $\sim 20 \AA$ and electron density $\sim 0.29 \mathrm{e} / \AA^{3}$ corresponding to a closely packed alkyl chain region. The integrated electron density of the headgroup region is $\sim 2.5 \mathrm{e} / \AA^{2}$ which includes contributions from the phosphate groups, adsorbed $\mathrm{Nd}^{3+}$ and water molecules. In the case of $\mathrm{Lu}^{3+}$, the ED profile suggests an inverted bilayer structure. However, the boxes do not appear to have one-to-one correspondence with the molecular structure. So, we perform an analysis of the ED profile as below.

We ascribe the peak in ED profile (at $z \sim-30 \AA$ ) for $\mathrm{Lu}^{3+}$-DHDP surface to a layer of $\mathrm{Lu}^{3+}$ ions (Figure $3 \mathrm{~d}$ ). For a regular bilayer, the electron density should uniformly decrease from this $\mathrm{Lu}^{3+}$ layer to the bulk aqueous region. The presence of a lower density region in the ED between this $\mathrm{Lu}^{3+}$ layer and the aqueous bulk region (ED 0.33) suggests the presence of alkane chains, which in turn indicates that the surface is covered with an inverted bilayer. There can be two canonical conformations for the inverted bilayer - two DHDP molecules exposed to the air side and one towards the aqueous side, or vice versa. We can decompose the ED profile shown in Figure 3(b) into three regions - top, middle, and bottom layers (Figure $3 \mathrm{~d}$ ). We assume that the DHDP tail groups are fully extended in both top and bottom layers. This assumption is supported by the absence of any gauche defects in the alkyl chains as shown by the VSFG spectra (Figure 2b). Thus, we make the top and bottom layers to have approximately $20 \AA$ thickness which corresponds to the full length of all-trans hexadecyl chains. This gives us an integrated electron density of $\sim 2.6 \mathrm{e} / \AA^{2}, 7.5 \mathrm{e} / \AA^{2}$, and $6.1 \mathrm{e} / \AA^{2}$ for top, middle, and bottom layers, respectively. The higher electron density of the bottom layer than the top layer suggests that there are more DHDP molecules adjacent to the aqueous side. This conformation is favored possibly due to the higher shielding of water from the chains with a tightly packed alkyl chain layer compared to a loosely packed alkyl chain region.

X-ray fluorescence near total reflection (XFNTR) spectroscopy probes the elemental composition of the interfacial region. Figure $S 2$ shows the variation of integrated fluorescence signal in the $L_{\alpha}$ region of $\mathrm{Nd}$ and $L u$ as a function of the vertical momentum transfer $\left(Q_{z}\right)$. The fits to the data provide an estimate of the interfacial number density of the elements (Table S1). For $\mathrm{Nd}^{3+}$ we obtain $\sim 116 \pm 4 \AA^{2}$ per Nd. At $\sim 40$ $\AA^{2}$ per DHDP molecule there is $\sim 0.34 \pm 0.01 \mathrm{Nd}^{3+}$ per DHDP which is close to the $\mathrm{Nd}^{3+}$ number density required for balancing the charge of the monolayer. With $L^{3+}$ however, we obtain $41 \pm 1 \AA^{2}$ per $L u$, or approximately 0.66 Lu per DHDP molecule at $27 \AA^{2}$ per DHDP. The minimum surface area per $\mathrm{Lu}^{3+}$ when both the top and bottom DHDP layers are fully packed is $1 / 2 * 120 \AA^{2}=60 \AA^{2}$. As the obtained surface area is significantly less than this minimum, the speciation of $L u$ is probably different from the trivalent species found in bulk solution, i.e. counterions may be present in the bilayer to compensate the charge.

\section{Lanthanide injection to subphase after DHDP monolayer formation - Kinetic studies}

In the previous section DHDP is spread on the lanthanide solutions. $\mathrm{Lu}^{3+}$ is a highly charge dense ion and it is likely to interact very strongly with the molecules of DHDP even before the DHDP molecules are compressed, i.e., when the molecules are in a 2-D gaseous state on the surface. So, while compression, 
we could be compressing a $\mathrm{Lu}^{3+}$-DHDP complexes instead of non-interacting DHDP molecules. We have conducted the following experiment (Figure 4) to study whether a preformed monolayer of DHDP spontaneously reorganizes into an inverted bilayer in the presence of $\mathrm{Lu}^{3+}$, resembling the structure described in the previous section.

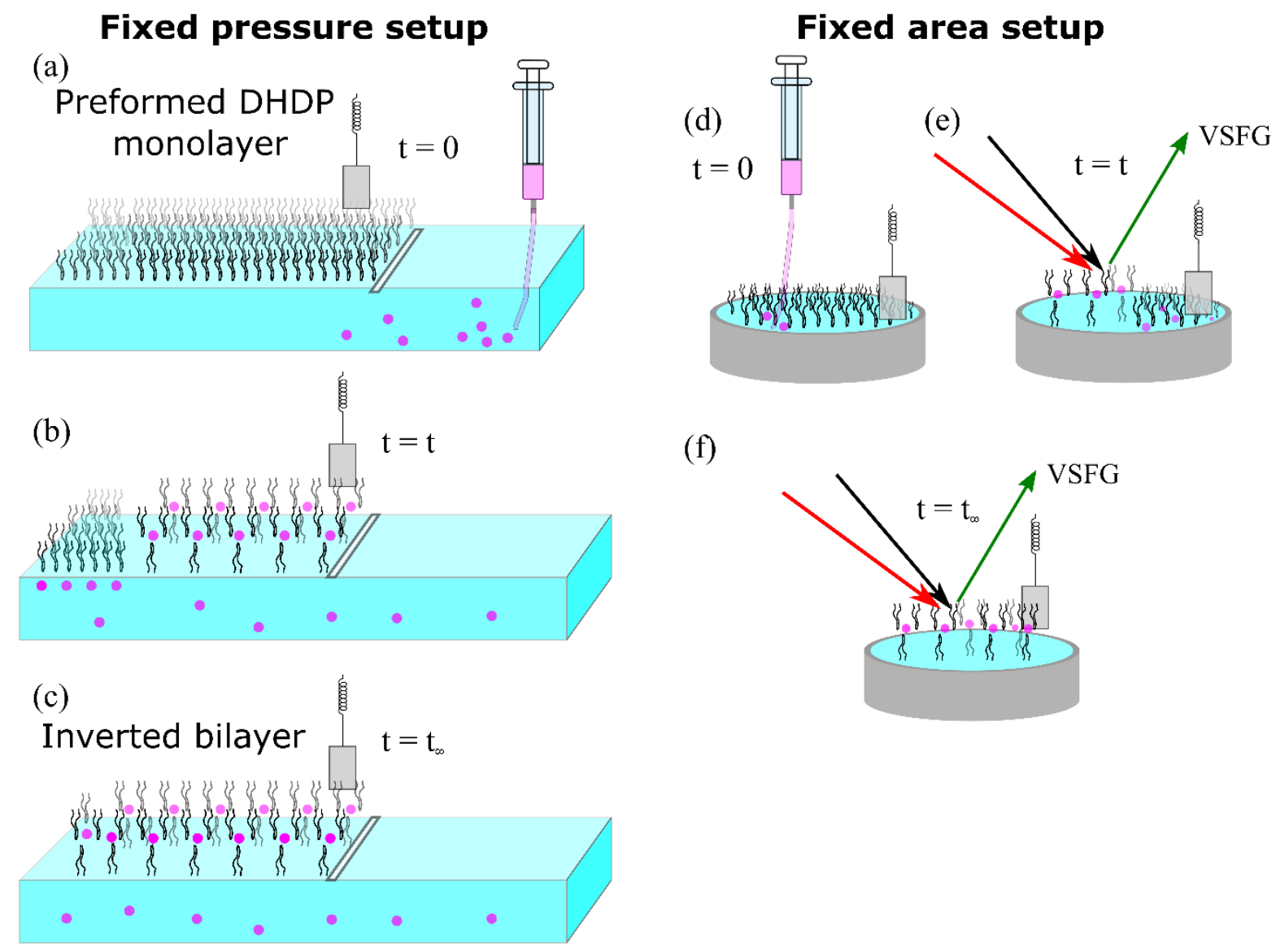

Figure 4. Schematic of kinetic study of changes in the interfacial structure of DHDP-covered surfaces upon introducing lanthanide ions in the subphase. (a) a monolayer of DHDP is spread on water, and lanthanide solution is injected behind the barrier $(t=0),(b)$ changes in the monolayer structure under constant pressure is monitored by changes in the barrier position as a function of time, (c) a (meta)stable state is reached at $\mathrm{t}=\mathrm{t}_{\infty}$ where the barrier position does not change. Changes in the interfacial structure were also monitored with VSFG spectroscopy in a fixed area setup- $(d)$ a monolayer of DHDP is spread on water and lanthanide solution is injected into the subphase $(t=0),(e)$ changes in the $\mathrm{CH}_{2}$ and $\mathrm{CH}_{3}$ spectral region are monitored as a function of time until a no change is detected ( $\mathrm{f}$ ).

XR of the interface after compressing DHDP on water to a surface pressure of $10 \mathrm{mN} / \mathrm{m}$ shows the formation of a monolayer (Figure 5). Upon introduction of $\mathrm{Lu}^{3+}$ ions in the subphase, behind the trough barrier, the X-ray reflectivity shows a gradual change along with decrease in surface area for maintaining constant surface pressure. About 4.5 hours after the introduction of $\mathrm{Lu}^{3+}$, the XR curve becomes very similar to the one shown in Figure 3(a) indicating that the interface has an inverted-bilayer structure similar to the one obtained by direct spreading of DHDP on $\mathrm{Lu}^{3+}$-containing subphase. We note that the 
timescale of XR measurement ( 45 minutes per scan) is of the order of kinetics of changes in interfacial structure. Thus, it is difficult to quantify the exact interfacial structures in the transient region $(<4 \mathrm{~h})$.

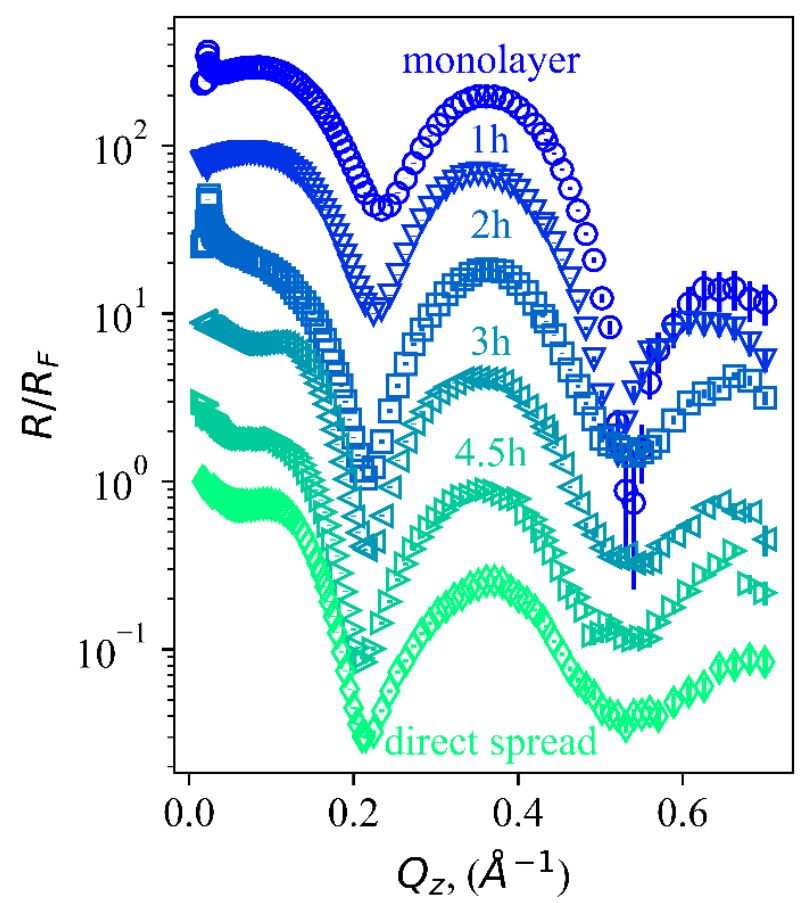

Figure 5. Time-dependent variation of the Fresnel-normalized XR profile of the DHDP-covered upon introduction of $\mathrm{Lu}^{3+}$ to the subphase. DHDP is spread on water and compressed to form a monolayer at a surface pressure of $10 \mathrm{mN} / \mathrm{m}$. At $\mathrm{t}=0$, a small volume of $\mathrm{LuCl}_{3}$ solution is injected into the subphase, behind the trough barrier. The monolayer begins to shrink in area while maintaining a constant surface pressure (see Figure 6 (a) for the surface pressure isotherm). After $\sim 4.5$ hours, the XR profile of the surface resembles that of DHDP spread directly on $\mathrm{LuCl}_{3}$ solution (Figure 3(a)). The XR curves at different time stamps have been shifted vertically for clarity.

The effect of monovalent anions on the interfacial adsorption and transport of lanthanides has been reported earlier. ${ }^{8-9,40}$ We found no effect of background ions on the interfacial structure when spreading DHDP on the subphase containing lanthanides and background salts (Figure S3). However, there is an anion-dependence in the kinetics of monolayer to inverted bilayer transition (Figure 6) upon addition of $\mathrm{Lu}^{3+}$. We spread DHDP monolayer on salt solutions ( $0.1 \mathrm{M}$ of $\mathrm{NaNO}_{3}$ or $\mathrm{NaSCN}$ ) and introduced either $\mathrm{Nd}^{3+}$ or $\mathrm{Lu}^{3+}$ solution behind the barrier (Figure $4 \mathrm{a}-\mathrm{c}$ ). The barrier position was allowed to move to maintain a constant surface pressure of $10 \mathrm{mN} / \mathrm{m}$, same as the one used in the previous section. With introduction of $\mathrm{Nd}^{3+}$ the surface area remains constant with time (Figure S4). Figure 6(a) shows the decay of trough area required to maintain the surface pressure constant upon injecting $\mathrm{Lu}^{3+}$ solution. We have modeled the decay as an exponential function of time according to Equation 1. This model has three parameters - $t_{0}$ (time offset after which the decay is exponential), $k$ (rate constant for exponential decay), and $a_{\infty}$ (the ratio of final to initial trough area). The fit parameters are listed in Table S3. The rate of decay roughly corresponds to the monolayer to inverted bilayer transition. Thus, there is an effect of 
background anion on the monolayer to inverted bilayer transition. Both nitrate and thiocyanate increase the rate of transition with thiocyanate having a stronger effect.
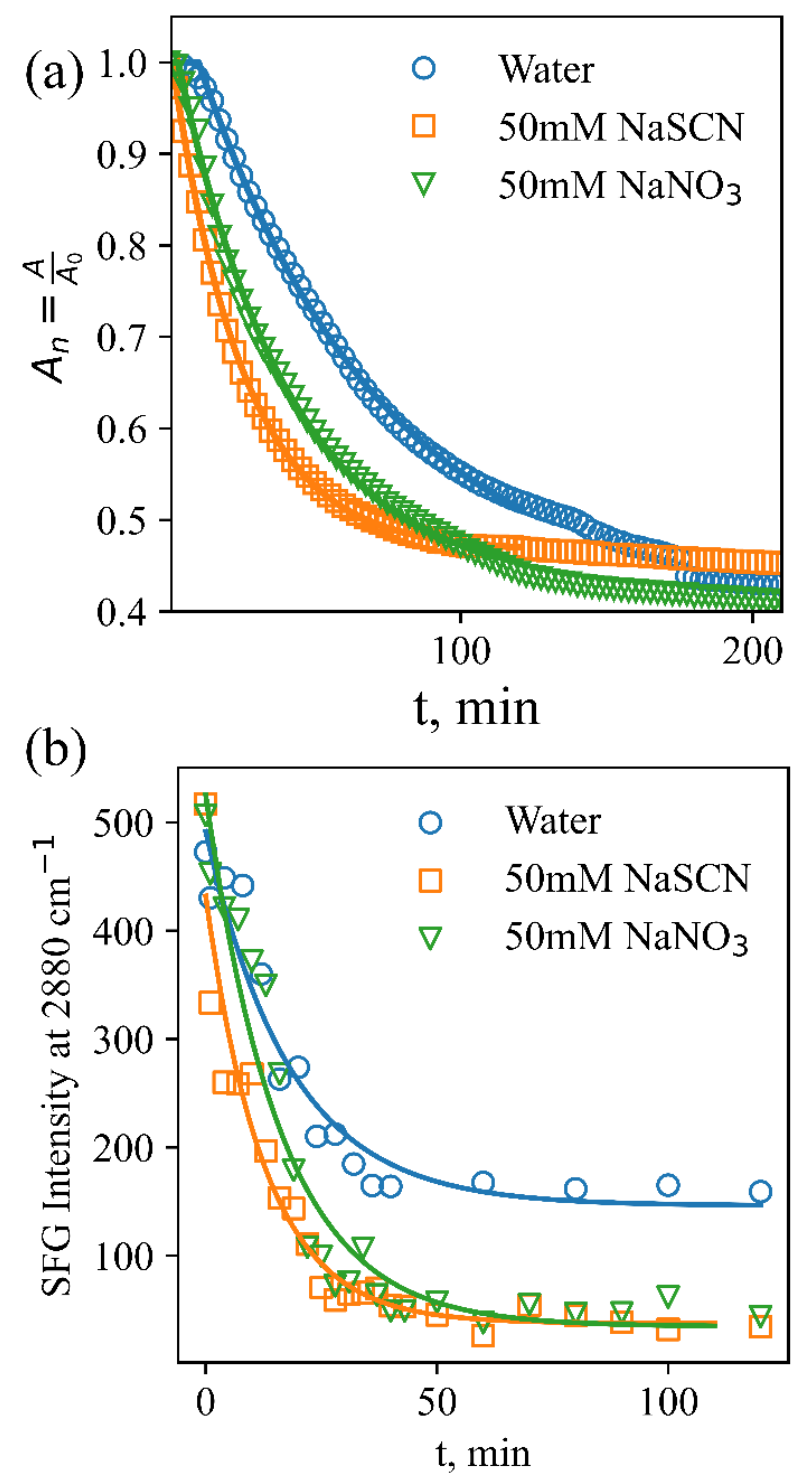

Figure 6. (a) The kinetics of the formation of the bilayer are dependent on the background anion present in the aqueous subphase. Thiocyanate (orange squares) leads to a faster formation of the bilayer compared to nitrate (green triangles), both faster than having no background salt (blue circles). The solid lines are fits to an empirical model based on nucleation growth theory shown in Eq. 1. (b) Decay rates of the amplitude of the $\mathrm{CH}_{3}$ symmetric stretch peak from VSFG experiments also indicate that the monolayer to inverted bilayer transition is faster in the presence of $\mathrm{NaSCN}$.

The intensity of $\mathrm{CH}_{3}$ symmetric stretch peak in VSFG spectra, at $2875 \mathrm{~cm}^{-1}$, also provides a measure of monolayer to bilayer transition. Figure $6 \mathrm{~b}$ shows the decay of this peak upon introduction of $\mathrm{Lu}^{3+}$ to the subphases below DHDP monolayers. An exponential decay model was used to fit the data (solid lines in Figure 6(b)) and the parameters are listed in Table S4. The rate of monolayer to inverted bilayer 
conversion is increased by background ions; and thiocyanate has a stronger effect than nitrate. The rate of conversion obtained from VSFG appears to be faster than corresponding rates obtained by surface pressure isotherms. There may be a few reasons for the difference. First, VSFG probes the molecular scale structure directly, as opposed to the macroscopic surface area measurement. Also, a constant area setup used in VSFG versus the constant pressure setup in the isotherms can contribute to the differences (Figure 4). We note that the intensity of $\mathrm{CH}_{3}$ symmetric stretch peak does not significantly change upon introducing $\mathrm{Nd}^{3+}$ ions to the subphase (Figure $\mathrm{S} 5$ ).

We also studied the temperature dependence of the monolayer to inverted bilayer transition in the absence of background salts (Figure 7a). The rate constant is derived from the fits to an empirical model based on the classical nucleation growth theory (Equation 1). Its variation as a function of the inverse temperature is shown in Figure 7b. The fit parameters are tabulated in Table S3. The linear relationship shows that the monolayer to inverted bilayer transition follows the Arrhenius equation. We obtain an activation energy of $\sim 40 \mathrm{kcal} / \mathrm{mol}$ for the transition. Induction time for monolayer to inverted bilayer transition $\left(\mathrm{t}_{0}\right)$ decreases with increasing temperature. 

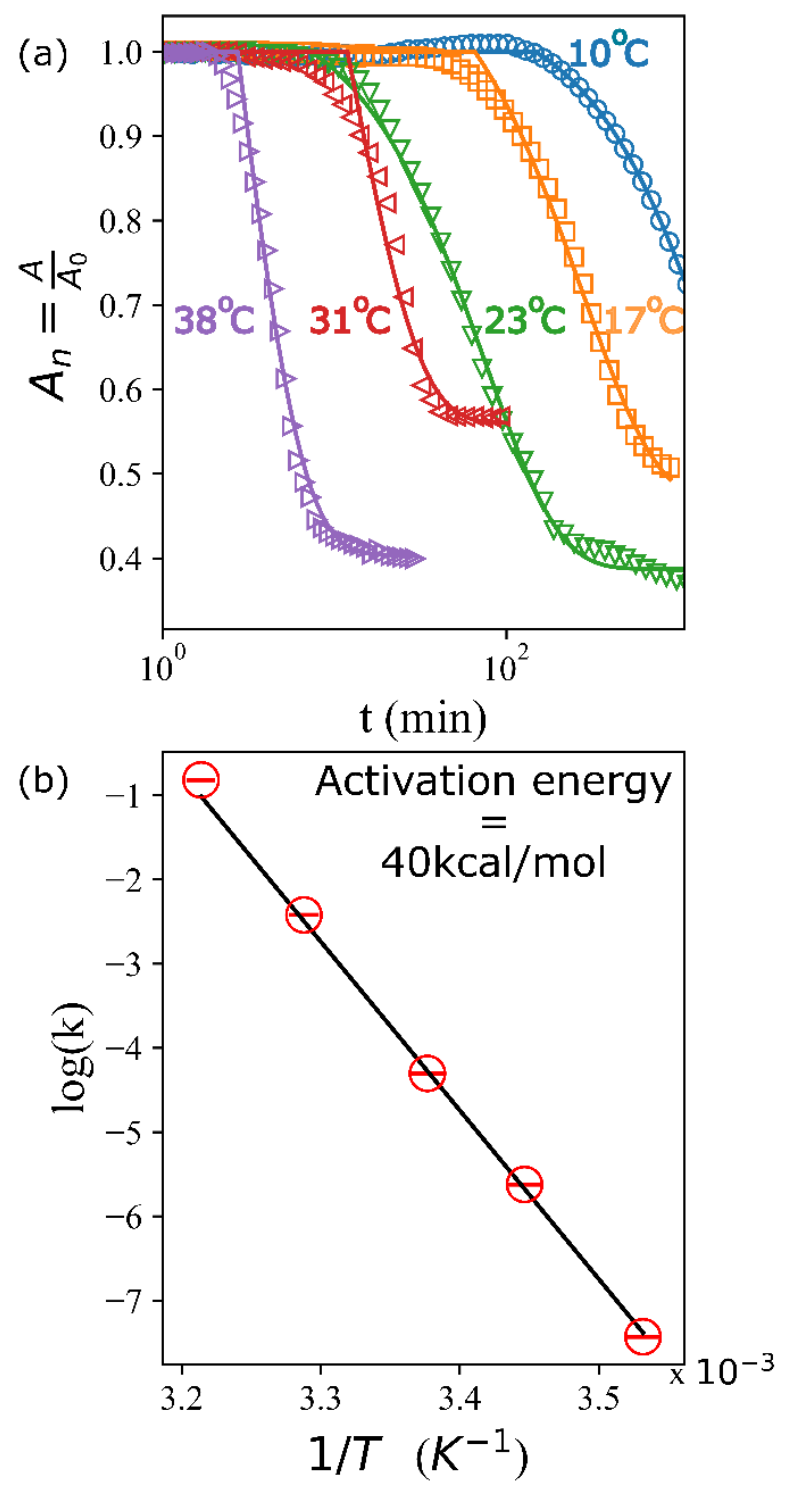

Figure 7. (a)Temperature dependence of the normalized area that is required to maintain a constant surface pressure of $10 \mathrm{mN} / \mathrm{m}$ as a function of time with injection of $\mathrm{LuCl}_{3}$ to the subphase (final concentration of $0.1 \mathrm{mM}$ ). While at a low temperature of $10^{\circ} \mathrm{C}$, the compression of surface due to the formation of the bilayer occurs in the scale of hours, at $38^{\circ} \mathrm{C}$ the transition occurs within minutes. Black solid lines show the fits obtained using an exponential decay, corresponding to first order kinetics of the transition (Eq. 1). (b) Variation of the first order rate constant (k) of monolayer-bilayer transition shows a classical Arrhenius type of behavior.

\section{DISCUSSIONS}

We have studied the differences in $\mathrm{Lu}^{3+}$ and $\mathrm{Nd}^{3+}$ adsorption onto DHDP-laden aqueous surfaces using $\mathrm{X}$ ray scattering, VSFG, and surface pressure isotherms. We find that, regardless of the surface preparation method, DHDP forms an inverted bilayer structure in the presence of $\mathrm{Lu}^{3+}$ ions and a monolayer in the presence of $\mathrm{Nd}^{3+}$ ions (Figure 8). Given the general chemical similarity among the lanthanides, this is an intriguing result showing the unique features of charged interfaces. 
(a)

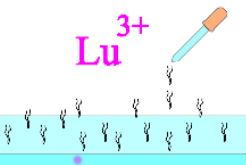

(b) Compression

to inv-bilayer

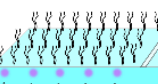

is $i \leqslant$ i

(c) Monolayer

to inv-bilayer

. M (d)

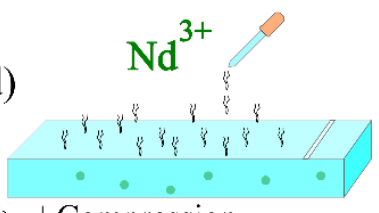

(e) $\mid$ Compression to monolayer

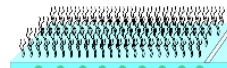

(f) $\uparrow$ Stable monolayer แ

Figure 8. DHDP molecules organize as an inverted bilayer in the presence of $\mathrm{Lu}^{3+}(\mathrm{b})$ both when spreading DHDP on $\mathrm{Lu}^{3+}$ containing subphase (a), and when injecting $\mathrm{Lu}^{3+}$ below a preformed DHDP monolayer. With $\mathrm{Nd}^{3+}$ however, DHDP forms a monolayer (e) regardless of the preparation method (d,f).

Interfacial adsorption and transport of lanthanide ions is of great research interest due to their relevance in rare earth element separations. ${ }^{1,4}$ Selectivity in rare earth extractions has been suggested to originate at the liquid-liquid interface due to electrostatic interactions. ${ }^{5}$ In the context of LLE, ionsurfactant interactions at the air/aqueous interface can elucidate both the dynamic ion transport across the interface and the equilibrium structures formed by the ion-extractant complexes in the organic phase. Dynamic structures forming at the liquid-liquid interface, such as water fingers, water ridges, and chemical "hinges" have been reported as some of the driving mechanisms for interfacial transport. ${ }^{41-45}$ Self-assembly of extractant-ion aggregates at the interface can also play a dynamic role in the interfacial ion transport. ${ }^{21}$ The ion-specific formation of inverted bilayer with $\mathrm{Lu}^{3+}$ reported here indicates a possible difference in dynamics of ion transfer with lighter and heavier lanthanides.

The effects of monovalent ions under dilute concentrations on charged monolayers are well described by the linearized Poisson-Boltzman equation except in the case of some highly polarizable ions. ${ }^{46-48}$ Theoretical models that treat the ions as point charges in a continuous medium are generally adequate but several recent reports show the importance of solvent restructuring near the interface. ${ }^{8,48-49}$ Even the apparent conformity of adsorption effects of most monovalent ions with the linearized PoissonBoltzmann theory has been suggested to be due to the cancellation of two non-classical terms. ${ }^{48}$ Multivalent ions can however induce "charge-reversal" or spontaneous collapse of monolayers which have not been reported with monovalent ions. ${ }^{50-51}$ In this context, effects of ion-ion correlation and ionmonolayer correlations have been described. ${ }^{52}$ Further, specific ion-monolayer interactions such as hydrogen bonding and coordination bonds can lead to ion-specific interactions at the interface. ${ }^{39,53}$ Rare earth ions are attractive candidates in probing multivalent ion-monolayer interactions as electrostatic interactions dominate their chemistry. ${ }^{54}$ Interactions of monolayers formed by amphiphiles with rare earth ions can shed light on their interactions with extractants and hence selectivity in LLE. All the rare earth ions are predominantly +3 charged and the main difference between them is in their decreasing ionic sizes with increasing atomic number, a phenomenon known as the lanthanide contraction. ${ }^{55-56}$

DHDP is not used as an extractant in commercial settings due to its poor solubility in alkanes. When using it as an interfacial analogue to HDEHP, it is important to consider the effects of tail structure. In 
LLE, it is well known that the tail group can play a major role in extraction, organic phase structure and the phase behavior. ${ }^{57-60}$ Similarly, the tail structure plays a major role in the formation and structure of the Langmuir monolayers due to the Van der Waals interactions between the tails. ${ }^{25,61-62}$ Our results with DHDP at air-water interface and the prior results obtained with DHDP at water-dodecane interface show that an inverted bilayer of DHDP is formed with heavy, charge dense trivalent ions. ${ }^{7,}$ At the dodecane-water interface, $\mathrm{Sr}^{2+}$ adsorbs to a monolayer of DHDP at the interface. ${ }^{6}$ This difference between trivalent and divalent ions was interpreted as different extraction mechanisms for the two ions with dialkyl phosphoric acid extractants. ${ }^{7}$ These experiments required lower temperatures to kinetically arrest the interfacial species formed during ion transfer. In contrast to the results obtained with DHDP, VSFG studies with HDEHP at the air-water interface suggest that HDEHP interacts with all the lanthanides in a monolayer-like structure. ${ }^{22,63}$ We note that studies with VSFG alone provide limited conclusions as the interpretation is convoluted by the third-order nonlinear effects and the molecular orientation at the surface. Similarly, X-ray reflectivity of the HDEHP at the air-water interface is not qualitatively different for light and heavy lanthanides. ${ }^{23}$ With a shorter chain analogue of HDEHP, namely dibutyl phosphate, a structure similar to inverted-bilayer is observed. ${ }^{63}$ Organic phase speciation of Er is also different when extracted with HDEHP (hexamer) or DHDP (trimer). ${ }^{24}$ Regardless of these differences, it is evident that the small changes in ionic size of the lanthanides can have a major implications to the organic phase structure and thereby the extraction energetics.

Although there are several studies on the collapse of Langmuir monolayers due to compression ${ }^{64-67}$ there are fewer on the spontaneous collapse of monolayers induced by solutes. Recently, a spontaneous monolayer to inverted bilayer transformation of palmitic acid in the presence of calcium ions has been reported. ${ }^{51}$ Divalent cations have also been shown to reduce the surface pressure at which stearic acid films collapse. ${ }^{68}$ Interplay of ion-lipid interactions is especially important in the biophysics of cellular membranes where lipids affect selective ion transport and ions affect transbilayer lipid motions. ${ }^{69-72} \mathrm{An}$ ion size-dependence on the flip-flop of the lipids in the bilayer has been reported..$^{73}$ In this context, interestingly, a coordination-driven, ion-specific monolayer to inverted bilayer transition has been reported with calix[4]arene films on $\mathrm{Cu}$ (II) or $\mathrm{Ni}(\mathrm{II})$ solutions. ${ }^{74}$ Unlike the lanthanide ions where ligands interact electrostatically, transition metals can show significant selectivities due to ligand-metal coordination bonds that are directional.

The kinetics of monolayer to inverted bilayer transition with $\mathrm{Lu}^{3+}$ in the subphase is strongly temperature dependent with an activation barrier of $\sim 40 \mathrm{kcal} / \mathrm{mol}$. This relatively large energy barrier is in correspondence with the lower temperature required to arrest the heavy lanthanide species at the dodecane-water interface. ${ }^{24}$ The onset of transition is also temperature dependent - at lower temperatures the transition starts later indicating a temperature dependent nucleation of inverted bilayer structure. DHDP forms stable monolayers with $\mathrm{Nd}^{3+}$ in the subphase at temperatures up to $37^{\circ} \mathrm{C}$. Whether inverted bilayers can form with lighter lanthanides at higher temperatures is an open question. It is not clear whether inverted bilayer formation is kinetically limited or whether the inverted bilayers are thermodynamically unstable with $\mathrm{Nd}^{3+}$.

We have previously reported the anion-dependence in lanthanide adsorption at DHDP monolayers. ${ }^{9}$ These experiments were conducted with preformed monolayers and lanthanide injection behind the barrier. Under the impression that the surfaces were under equilibrium, we attributed the differences in fluorescence signals to anion-effect on number density of lanthanides adsorbing to the surface. We 
obtained an interfacial area per Lu that is lower than that for 1:3 Lu:DHDP complexes and interpreted that to be a signature of presence of background anions next to the DHDP monolayer. Our current work shows that the lower interfacial area per Lu is obtained even in the absence of excess background salts. Further, the reported difference in Lu adsorption with different background salts are likely due to the differences in kinetics of inverted bilayer formation in the presence of $\mathrm{NO}_{3}{ }^{-}$and $\mathrm{SCN}^{-}$.

\section{CONCLUSIONS}

We have demonstrated a convenient model system to study the ion-amphiphile interactions which are particularly important in ion recognition and selectivity in LLE. Phosphoric acid based extractants are often used in the LLE of rare earth elements, but there are outstanding questions on the mechanisms that determine ion transport and selectivity. Here we have shown that there is an ion-specific effect in the adsorption of trivalent ions to DHDP, an analogue of phosphoric acid based extractants: larger trivalent ion $\mathrm{Nd}^{3+}$ maintains the monolayer structure of DHDP while smaller $\mathrm{Lu}^{3+}$ ion induces the formation of an inverted bilayer. This shows that the air/aqueous interface models can be used to reproduce important aspects of oil/water interfaces. Further, the monolayer of DHDP collapses to an inverted bilayer upon the introduction of $\mathrm{Lu}^{3+}$ ions where the rate of collapse is temperature dependent. These results show that the minor differences in ionic sizes of the rare earth elements have major impacts on the interfacial structure. Elucidating these effects at the air-water interface, a much simpler system compared to the organic phase structures in LLE, can show the drivers of ion-transport and selectivity in LLE. The kinetics of ion-dependent monolayer to inverted bilayer transition provide a route to investigate ion transport and lipid structure in various physicochemical processes.

\section{EXPERIMENTAL METHODS}

Materials: Dihexadecyl phosphate (DHDP) was purchased from Avanti Polar Lipids Inc. Neodymium (III) chloride hexahydrate, lutetium (III) chloride hexahydrate, sodium chloride, sodium nitrate, and sodium thiocynate were purchased from Sigma Aldrich Inc., and used as received without any further purification.

Aqueous solutions of lanthanides and sodium salts were prepared in volumetric flasks. DHDP was dissolved in chloroform to prepare a $0.25 \mathrm{mM}$ solution.

Sample preparation for static studies: X-ray scattering studies were conducted at sector 15-ID-C of Advanced Photon Source, Argonne National Laboratory. In a typical experiment the aqueous subphase containing lanthanides was poured into the Langmuir trough placed in a sealable chamber. The liquid surface was compressed and aspirated as a surface preparation step. With the barrier in the "open" position, a measured volume of DHDP solution was spread on the surface, dropwise, with a $100 \mu \mathrm{L}$ glass Hamilton syringe. The surface was then compressed by moving the motorized barrier to reach a surface pressure of $10 \mathrm{mN} / \mathrm{m}$. The surface is kept under this constant pressure by barrier motion controlled with a NIMA IU4 controller. The chamber was then sealed and purged with water-saturated He gas.

The VSFG studies were conducted in a fixed area mode. Approximately $40 \mathrm{~mL}$ of the aqueous subphase is poured into a shallow PTFE dish with circular cross-section (diameter $=10 \mathrm{~cm}$ ). Droplets of DHDP solution were spread on the surface of the liquid slowly using $10 \mu \mathrm{L}$ Hamilton syringe while monitoring the surface pressure. Enough droplets were added to achieve a surface pressure of $10 \mathrm{mN} / \mathrm{m}$. 
Sample preparation for kinetic studies: For X-ray scattering experiments $198 \mathrm{~mL}$ of lanthanide-free aqueous subphase (water or a salt solution) was poured into the trough and DHDP was spread on the liquid surface. The monolayer was compressed to a surface pressure of $10 \mathrm{mN} / \mathrm{m}$. After the formation of a stable monolayer $2 \mathrm{~mL}$ of the lanthanide solution at an appropriate concentration was added to the subphase by injecting the solution behind the trough barrier using a plastic syringe. The subphase was then mixed by pumping the solution in and out of the syringe for 10 minutes.

For temperature dependent kinetic studies the trough temperature was controlled by pumping an ethylene glycol solution ( $\sim 5 \mathrm{wt} \%$ ) that was cooled or heated with a NESLAB RTE 111 chiller. The temperature of the subphase was measured using a thermocouple.

We used the following empirical model based on the classical nucleation-growth theory ${ }^{75}$ to fit the decay of trough area required to hold a constant surface pressure in the presence of $\mathrm{Lu}^{3+}$ ions as follows:

$$
\frac{a(t)-a_{\infty}}{1-a_{\infty}}=u\left(t-t_{0}\right)\left(e^{-k\left(t-t_{0}\right)}\right)+u\left(t_{0}-t\right) \text { Eq.1 }
$$

Where $\mathrm{a}(\mathrm{t})$ is the ratio of area at a given time to the initial monolayer area, $\mathrm{k}$ is the exponential decay rate constant, $\mathrm{t}_{0}$ is the time delay between the introduction of $\mathrm{Lu}^{3+}$ and the onset of exponential decay, and $\mathrm{a}_{\infty}$ is the area ratio at steady state, and $u(x)$ is the Heaviside function.

Synchrotron X-ray studies: XR and XFNTR studies were conducted at sector 15-ID-C of Advanced Photon Source, Argonne National Laboratory as described in our previous papers. ${ }^{8,}{ }^{34} \mathrm{X}$-ray energy was set to $18.3 \mathrm{keV}$, above the L3 absorption edges of Lu and Nd. Dectris PILATUS 100 detector was used for obtaining reflected $X$-ray intensity. At each incident angle, a background signal was subtracted from the reflection. The XR data was fit using Stochfit. ${ }^{76}$ XFNTR data was collected using an energy dispersive Vortex detector setup normal to the surface with a collection snout placed $\sim 10.6 \mathrm{~mm}$ from the surface. At each incidence angle, Gaussian curves were fit to the fluorescence spectra corresponding to the element of interest. Integrals of the peaks are used in the XFNTR figures.

VSFG Experiments: The VSFG measurements are acquired using an EKSPLA system, which has been described previously. ${ }^{8,10-11,34}$ Briefly, an amplified Nd:YAG laser system produces 29 ps pulses having 28 $\mathrm{mJ}$ energy centered at $1064 \mathrm{~nm}$ with a repetition rate of $50 \mathrm{~Hz}$. The harmonic unit splits the $1064 \mathrm{~nm}$ laser and generates two beams of $532 \mathrm{~nm}$. Fundamental laser beam $1064 \mathrm{~nm}$ and second harmonic beam $532 \mathrm{~nm}$ are used to generate a narrowband IR pulse tunable from $1000-4000 \mathrm{~cm}^{-1}$ via optical parametric generator and difference frequency generation. The other $532 \mathrm{~nm}$ laser beam is used as a visible source to generate VSFG signal. The SFG signal is passed through a monochromator and collected using a photomultiplier tube.

The VSFG measurement is carried out using a reflection geometry where the incident angles of the visible and IR beams are $60^{\circ}$ and $55^{\circ}$, respectively, to the surface normal. The visible light is attenuated to an average energy of $600 \mu \mathrm{J}$ and the IR energy is maintained at $100 \mu \mathrm{J}$ for all measurements. A motorized piezoelectric rotation stage is used to rotate the sample to avoid beam damage. Each spectrum is collected with a $4 \mathrm{~cm}^{-1}$ increment over the range of $2800-3800 \mathrm{~cm}^{-1}$ and averaged over 300 laser shots per point. The spectra are collected under various polarization combinations and are normalized against the SFG spectrum of a z-cut quartz.

\section{AUTHOR INFORMATION}




\section{Corresponding Author}

*Email: ahmet@anl.gov. Web: www.anl.gov/profile/ahmet-uysal

\section{Author Contributions}

The manuscript was written through contributions of all authors. All authors have given approval to the final version of the manuscript.

Notes

Authors declare no competing financial interest.

\section{ACKNOWLEDGEMENTS}

We thank Wei Bu for his help with the liquid surface synchrotron experiments. The work presented here is supported by the U.S. Department of Energy, Office of Basic Energy Science, Division of Chemical Sciences, Geosciences, and Biosciences, Separation Science program under contract DE-ACO206CH11357. Use of the Advanced Photon Source, an Office of Science User Facility operated for the U.S. Department of Energy (DOE) Office of Science by Argonne National Laboratory, was supported by the U.S. DOE under Contract No. DE-AC02-06CH11357. NSF's ChemMatCARS Sector 15 is principally supported by the Divisions of Chemistry (CHE) and Materials Research (DMR), National Science Foundation, under Grant NSF/CHE-1834750.

\section{ABBREVIATIONS}

XFNTR, X-ray fluorescence near total reflection; VSFG, vibrational sum frequency generation; LLE, liquidliquid extraction; XR, X-ray reflectivity; DHDP, Dihexadecylphosphate;

\section{ASSOCIATED CONTENT}

\section{SUPPORTING INFORMATION.}

The supporting information document has the following: a) VSFG spectra showing the $\mathrm{OH}$ region, b) results from X-ray fluorescence near total reflection, c) X-ray reflectivity curves in the presence of salts, d) Surface pressure isotherm of DHDP on $\mathrm{Nd}^{3+}$ solution showing its stability, and e) Fit parameters for the empirical model describing the kinetics of monolayer to inverted bilayer transition.

REFERENCES

1. Moyer, B., Changing the landscape in solvent extraction. 1st ed.; CRC Press: Boca Raton, 2020; Vol. 23, p 310.

2. Wilson, A. M.; Bailey, P. J.; Tasker, P. A.; Turkington, J. R.; Grant, R. A.; Love, J. B., Solvent extraction: the coordination chemistry behind extractive metallurgy. Chem. Soc. Rev. 2014, 43, 123-34.

3. Nelson, J. J. M.; Schelter, E. J., Sustainable Inorganic Chemistry: Metal Separations for Recycling. Inorg. Chem. 2019, 58, 979-990.

4. Clark, A. E., Amphiphile-Based Complex Fluids: The Self-Assembly Ensemble as Protagonist. ACS Cent. Sci. 2019, 5, 10-12. 
5. Miller, M.; Liang, Y.; Li, H.; Chu, M.; Yoo, S.; Bu, W.; Olvera de la Cruz, M.; Dutta, P., Electrostatic Origin of Element Selectivity during Rare Earth Adsorption. Phys. Rev. Lett. 2019, 122, 058001.

6. $\quad$ Bu, W.; Mihaylov, M.; Amoanu, D.; Lin, B. H.; Meron, M.; Kuzmenko, I.; Soderholm, L.; Schlossman, M. L., X-ray Studies of Interfacial Strontium-Extractant Complexes in a Model Solvent Extraction System. J. Phys. Chem. B 2014, 118, 12486-12500.

7. Liang, Z.; Bu, W.; Schweighofer, K. J.; Walwark, D. J.; Harvey, J. S.; Hanlon, G. R.; Amoanu, D.; Erol, C.; Benjamin, I.; Schlossman, M. L., Nanoscale view of assisted ion transport across the liquid-liquid interface. Proc. Natl. Acad. Sci. U.S.A 2019, 116, 18227-18232.

8. Lovering, K.; Nayak, S.; Bu, W.; Uysal, A., The Role of Specific lon Effects in Ion Transport: The Case of Nitrate and Thiocyanate. J. Phys. Chem. C 2020, 124, 573-581.

9. Nayak, S.; Lovering, K.; Bu, W.; Uysal, A., Anions Enhance Rare Earth Adsorption at Negatively Charged Surfaces. J. Phys. Chem. Lett. 2020, 11, 4436-4442.

10. Nayak, S.; Kumal, R. R.; Liu, Z.; Qiao, B.; Clark, A. E.; Uysal, A., Origins of Clustering of MetalateExtractant Complexes in Liquid-Liquid Extraction. ACS Appl. Mater. Interfaces 2021, 13, 24194-24206.

11. Rock, W.; Qiao, B. F.; Zhou, T. C.; Clark, A. E.; Uysal, A., Heavy Anionic Complex Creates a Unique Water Structure at a Soft Charged Interface. J. Phys. Chem. C 2018, 122, 29228-29236.

12. Uysal, A.; Rock, W.; Qiao, B. F.; Bu, W.; Lin, B. H., Two-Step Adsorption of PtCl62- Complexes at a Charged Langmuir Monolayer: Role of Hydration and Ion Correlations. J. Phys. Chem. C 2017, 121, 25377-25383.

13. Scoppola, E.; Watkins, E. B.; Campbell, R. A.; Konovalov, O.; Girard, L.; Dufreche, J. F.; Ferru, G.; Fragneto, G.; Diat, O., Solvent Extraction: Structure of the Liquid-Liquid Interface Containing a Diamide Ligand. Angew. Chem., Int. Ed. 2016, 55, 9326-30.

14. Zhou, N. F.; Neuman, R. D., Dihexadecyl Phosphate Monolayers at the Heptane Water Interface Model Interfacial System for Solvent-Extraction Studies. Colloids Surf. 1992, 63, 201-207.

15. Li, D. Q., Development course of separating rare earths with acid phosphorus extractants: A critical review. J. Rare Earths 2019, 37, 468-486.

16. Nash, K. L., The Chemistry of TALSPEAK: A Review of the Science. Solvent Extr. Ion. Exc. 2015, 33, $1-55$.

17. Neuman, R. D.; Zhou, N. F.; Wu, J. G.; Jones, M. A.; Gaonkar, A. G.; Park, S. J.; Agrawal, M. L., General-Model for Aggregation of Metal-Extractant Complexes in Acidic Organophosphorus SolventExtraction Systems. Sep. Sci. Technol. 1990, 25, 1655-1674.

18. Ibrahim, T. H., An Overview of the Physiochemical Nature of Metal-Extractant Species in Organic Solvent/Acidic Organophosphorus Extraction Systems. Sep. Sci. Technol. 2011, 46, 2157-2166.

19. Steytler, D. C.; Jenta, T. R.; Robinson, B. H.; Eastoe, J.; Heenan, R. K., Structure of reversed micelles formed by metal salts of bis(ethylhexyl) phosphoric acid. Langmuir 1996, 12, 1483-1489.

20. Grimes, T. S.; Jensen, M. P.; Debeer-Schmidt, L.; Littrell, K.; Nash, K. L., Small-angle neutron scattering study of organic-phase aggregation in the TALSPEAK process. J. Phys. Chem. B 2012, 116, 13722-30.

21. Chowdhury, A. U.; Lin, L.; Doughty, B., Hydrogen-Bond-Driven Chemical Separations: Elucidating the Interfacial Steps of Self-Assembly in Solvent Extraction. ACS Appl. Mater. Interfaces 2020, 12, 3211932130.

22. Kusaka, R.; Watanabe, M., Stoichiometry of Lanthanide-Phosphate Complexes at the Water Surface Studied Using Vibrational Sum Frequency Generation Spectroscopy and DFT Calculations. J. Phys. Chem. B 2021, 125, 6727-6731.

23. Sun, P.; Binter, E. A.; Liang, Z.; Brown, M. A.; Gelis, A. V.; Benjamin, I.; Bera, M. K.; Lin, B.; Bu, W.; Schlossman, M. L., Antagonistic Role of Aqueous Complexation in the Solvent Extraction and Separation of Rare Earth lons. ChemRxiv 2021, doi:10.33774/chemrxiv-2021-hr2n9. 
24. Bu, W.; Yu, H.; Luo, G.; Bera, M. K.; Hou, B.; Schuman, A. W.; Lin, B.; Meron, M.; Kuzmenko, I.; Antonio, M. R.; Soderholm, L.; Schlossman, M. L., Observation of a rare earth ion-extractant complex arrested at the oil-water interface during solvent extraction. J. Phys. Chem. B 2014, 118, 10662-74.

25. Kaganer, V. M.; Mohwald, H.; Dutta, P., Structure and phase transitions in Langmuir monolayers. Rev. Mod. Phys. 1999, 71, 779-819.

26. Elderdfi, M.; Sikorski, A. F., Langmuir-monolayer methodologies for characterizing protein-lipid interactions. Chem. Phys. Lipids 2018, 212, 61-72.

27. Ahlers, M.; Muller, W.; Reichert, A.; Ringsdorf, H.; Venzmer, J., Specific Interactions of Proteins with Functional Lipid Monolayers - Ways of Simulating Biomembrane Processes. Angew. Chem., Int. Ed. 1990, 29, 1269-1285.

28. Dynarowicz-Latka, P.; Dhanabalan, A.; Oliveira, O. N., Jr., Modern physicochemical research on Langmuir monolayers. Adv. Colloid Interface Sci. 2001, 91, 221-93.

29. Giner-Casares, J. J.; Brezesinski, G.; Mohwald, H., Langmuir monolayers as unique physical models. Curr. Opin. Colloid. Interface Sci. 2014, 19, 176-182.

30. Stefaniu, C.; Brezesinski, G., X-ray investigation of monolayers formed at the soft air/water interface. Curr. Opin. Colloid. Interface Sci. 2014, 19, 216-227.

31. Sung, W.; Kim, D.; Shen, Y. R., Sum-frequency vibrational spectroscopic studies of Langmuir monolayers. Curr. Appl. Phys. 2013, 13, 619-632.

32. Vollhardt, D., Brewster angle microscopy: A preferential method for mesoscopic

characterization of monolayers at the air/water interface. Curr. Opin. Colloid. Interface Sci. 2014, 19, 183-197.

33. Eisenthal, K. B., Liquid interfaces probed by second-harmonic and sum-frequency spectroscopy. Chem. Rev. 1996, 96, 1343-1360.

34. Kumal, R.; Nayak, S.; Bu, W.; Uysal, A., Chemical Potential Driven Reorganization of Anions between Stern and Diffuse Layers at the Air/Water Interface. ChemRxiv 2021, doi:10.33774/chemrxiv2021-f20gf-v2

35. Wang, W. J.; Park, R. Y.; Meyer, D. H.; Travesset, A.; Vaknin, D., lonic Specificity in pH Regulated Charged Interfaces: Fe3+ versus La3+. Langmuir 2011, 27, 11917-11924.

36. Zhuang, X.; Miranda, P. B.; Kim, D.; Shen, Y. R., Mapping molecular orientation and conformation at interfaces by surface nonlinear optics. Phys. Rev. B 1999, 59, 12632-12640.

37. Bera, M. K.; Bu, W.; Uysal, A., Liquid Surface X-Ray Scattering. In Physical Chemistry of Gas-Liquid Interfaces, Elsevier: 2018; pp 167-194.

38. Pershan, P. S.; Schlossman, M., Liquid surfaces and interfaces: synchrotron x-ray methods. Cambridge University Press: 2012.

39. Wang, W.; Park, R. Y.; Meyer, D. H.; Travesset, A.; Vaknin, D., lonic specificity in pH regulated charged interfaces: Fe3+ versus La3+. Langmuir 2011, 27, 11917-24.

40. Nayak, S.; Lovering, K.; Uysal, A., lon-specific clustering of metal-amphiphile complexes in rare earth separations. Nanoscale 2020, 12, 20202-20210.

41. Kikkawa, N.; Wang, L.; Morita, A., Microscopic Barrier Mechanism of Ion Transport through Liquid-Liquid Interface. J. Am. Chem. Soc. 2015, 137, 8022-5.

42. Wen, B.; Sun, C.; Zheng, W.; Bai, B.; Lichtfouse, E., Evidence for water ridges at oil-water interfaces: implications for ion transport. Soft Matter 2020, 16, 826-832.

43. Qiao, B.; Muntean, J. V.; Olvera de la Cruz, M.; Ellis, R. J., Ion Transport Mechanisms in LiquidLiquid Interface. Langmuir 2017, 33, 6135-6142.

44. Benjamin, L., Mechanism and dynamics of ion transfer across a liquid-liquid interface. Science 1993, 261, 1558-60. 
45. Liu, Z.; Clark, A. E., An octanol hinge opens the door to water transport. Chem. Sci. 2020, 12, 2294-2303.

46. Claesson, P.; Carmonaribeiro, A. M.; Kurihara, K., Dihexadecyl Phosphate Monolayers - Intralayer and Interlayer Interactions. J. Phys. Chem. 1989, 93, 917-922.

47. Bu, W.; Vaknin, D.; Travesset, A., How accurate is Poisson-Boltzmann theory for monovalent ions near highly charged interfaces? Langmuir 2006, 22, 5673-81.

48. Leontidis, E., Investigations of the Hofmeister series and other specific ion effects using lipid model systems. Adv. Colloid Interface Sci. 2017, 243, 8-22.

49. Marcus, Y., Effect of ions on the structure of water: structure making and breaking. Chem. Rev. 2009, 109, 1346-70.

50. Miller, M.; Chu, M.; Lin, B.; Meron, M.; Dutta, P., Observation of Ordered Structures in Counterion Layers near Wet Charged Surfaces: A Potential Mechanism for Charge Inversion. Langmuir 2016, 32, 73-7.

51. Zhang, P.; Pham, T.; Zheng, X.; Liu, C.; Plata, P. L.; Kral, P.; Bu, W.; Lin, B.; Liu, Y., Spontaneous collapse of palmitic acid films on an alkaline buffer containing calcium ions. Colloids Surf. B 2020, 193, 111100.

52. Travesset, A.; Vaknin, D., Bjerrum pairing correlations at charged interfaces. Europhys. Lett. 2006, 74, 181-187.

53. Sung, W.; Vaknin, D.; Kim, D., Different Adsorption Behavior of Rare Earth and Metallic lon Complexes on Langmuir Mono layers Probed by Sum-Frequency Generation Spectroscopy. J. Opt. Soc. Korea 2013, 17, 10-15.

54. Wilson, R. E.; Carter, T. J.; Autillo, M.; Stegman, S., Thiocyanate complexes of the lanthanides, Am and Cm. Chem. Commun. 2020, 56, 2622-2625.

55. Cotton, S. A.; Raithby, P. R., Systematics and surprises in lanthanide coordination chemistry. Coord. Chem. Rev. 2017, 340, 220-231.

56. Seitz, M.; Oliver, A. G.; Raymond, K. N., The lanthanide contraction revisited. J. Am. Chem. Soc. 2007, 129, 11153-11160.

57. Servis, M. J.; Wu, D. T.; Shafer, J. C., The role of solvent and neutral organophosphorus extractant structure in their organization and association. J. Mol. Liq. 2018, 253, 314-325.

58. Dul, M. C.; Braibant, B.; Dourdain, S.; Pellet-Rostaing, S.; Bourgeois, D.; Meyer, D., Perfluoroalkyl- vs alkyl substituted malonamides: Supramolecular effects and consequences for extraction of metals. J. Fluorine. Chem. 2017, 200, 59-65.

59. Rao, P. R. V.; Kolarik, Z., A review of third phase formation in extraction of actinides by neutral organophosphorus extractants. Solvent Extr. Ion. Exc. 1996, 14, 955-993.

60. Kolarik, Z., Review: Dissociation, Self-Association, and Partition of Monoacidic Organophosphorus Extractants. Solvent Extr. Ion. Exc. 2010, 28, 707-763.

61. Petrov, J. G.; Pfohl, T.; Mohwald, H., Ellipsometric chain length dependence of fatty acid Langmuir monolayers. A heads-and-tails model. J. Phys. Chem. B 1999, 103, 3417-3424.

62. Li, M. Y.; Acero, A. A.; Huang, Z. Q.; Rice, S. A., Formation of an Ordered Langmuir Monolayer by a Nonpolar Chain Molecule. Nature 1994, 367, 151-153.

63. Kusaka, R.; Watanabe, M., The structure of a lanthanide complex at an extractant/water interface studied using heterodyne-detected vibrational sum frequency generation. Phys. Chem. Chem. Phys. 2018, 20, 2809-2813.

64. Phan, M. D.; Lee, J.; Shin, K., Collapsed States of Langmuir Monolayers. J. Oleo. Sci. 2016, 65, 385-97.

65. Lee, K. Y., Collapse mechanisms of Langmuir monolayers. Annu. Rev. Phys. Chem. 2008, 59, 77191. 
66. Bu, W.; Vaknin, D., Bilayer and trilayer crystalline formation by collapsing behenic acid monolayers at gas/aqueous interfaces. Langmuir 2008, 24, 441-7.

67. Vaknin, D.; Bu, W.; Satija, S. K.; Travesset, A., Ordering by collapse: formation of bilayer and trilayer crystals by folding Langmuir monolayers. Langmuir 2007, 23, 1888-97.

68. Das, K.; Sah, B. K.; Kundu, S., Cation-induced monolayer collapse at lower surface pressure

follows specific headgroup percolation. Phys. Rev. E 2017, 95, 022804.

69. Heimburg, T., Lipid ion channels. Biophys. Chem. 2010, 150, 2-22.

70. Redondo-Morata, L.; Giannotti, M. I.; Sanz, F., Structural impact of cations on lipid bilayer models: nanomechanical properties by AFM-force spectroscopy. Mol. Membr. Biol. 2014, 31, 17-28.

71. Matsuzaki, K.; Yoneyama, S.; Murase, O.; Miyajima, K., Transbilayer transport of ions and lipids coupled with mastoparan X translocation. Biochemistry 1996, 35, 8450-6.

72. Lin, J.; Dargazany, R.; Alexander-Katz, A., Lipid Flip-Flop and Pore Nucleation on Zwitterionic Bilayers are Asymmetric under lonic Imbalance. Small 2017, 13.

73. Ma, Y. H.; Li, B. L.; Yang, J. J.; Han, X. F.; Chen, Z.; Lu, X. L., Metal lon Size-Dependent Effects on Lipid Transmembrane Flip-Flop. J. Phys. Chem. C 2019, 123, 17899-17907.

74. Moradi, M.; Lengweiler, N. L.; Housecroft, C. E.; Tulli, L. G.; Stahlberg, H.; Jung, T. A.;

Shahgaldian, P., Coordination-Driven Monolayer-to-Bilayer Transition in Two-Dimensional Metal-Organic Networks. J. Phys. Chem. B 2021, 125, 4204-4211.

75. Vollhardt, D.; Retter, U., Nucleation in Insoluble Monolayers .1. Nucleation and Growth-Model for Relaxation of Metastable Monolayers. J. Phys. Chem. 1991, 95, 3723-3727.

76. Danauskas, S. M.; Li, D. X.; Meron, M.; Lin, B. H.; Lee, K. Y. C., Stochastic fitting of specular X-ray reflectivity data using StochFit. J. App. Crystallogr. 2008, 41, 1187-1193. 


\section{$\underline{\text { TOC Figure }}$}

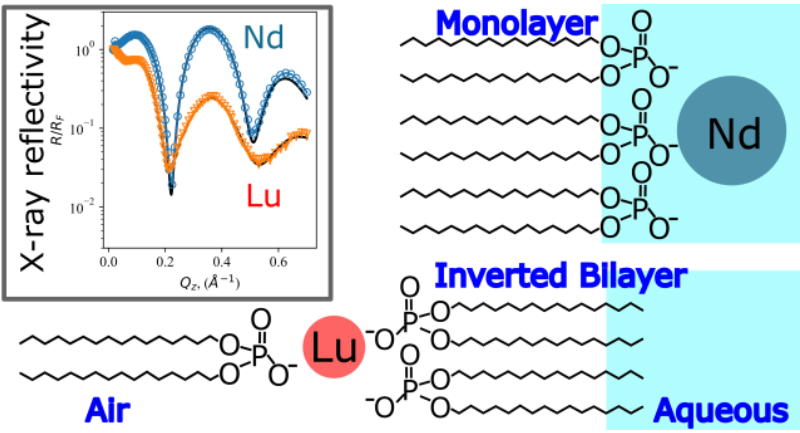

